\title{
DISEÑO: EXCUSA PARA UN COMENTARIO SOBRE EPISTEMOLOGÍA
}

\section{DESIGN. AN EXCUSE FOR A COMMENT ON EPISTEMOLOGY}

Por:

\section{Alberto Romero}

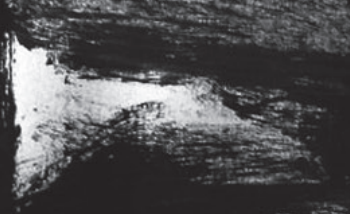

Profesor de la Facultad de Diseño Industrial Universidad Jorge Tadeo Lozano alberto.romero@utadeo.edu.co

Resumen: El siguiente texto tiene como propósito dialogar con un fragmento del pensamiento de Michel Foucault y orientar el diálogo hacia la formulación de preguntas sobre la fundamentación epistemológica y la noción de diseño.

El propósito central del texto es el de inspeccionar, desde el ejercicio metódico sobre la formación del discurso, la noción de épistéme y sus valores constitutivos como eje y fundamento de la comprensión y la instauración de las formas de análisis particulares al diseño; entendida la épistéme como territorio de relaciones y como lugar de múltiples discursos. Además, se pretende vincular la mencionada inspección, así como el propósito localizador y ordenador de la noción de discurso con su funcionamiento e institucionalización. Esto, en el marco de la reflexión sobre la función política del intelectual, en el que, pensar las disciplinas e intentar reconstruir las épistémes sería ya una función política.

Palabras Clave: Diseño, epistemología, disciplina. 
Abstract: The following is intended to create a dialogue with a fragment of the thought of Michel Foucault, and guide the dialogue towards the formulation of questions about the epistemological support and the notion of design.

The central purpose of the text is to inspect, from the methodical exercise on the formation of discourse, the notion of episteme and its constituent values as the core and base of understanding and establishment of forms to the design particular analysis; understood episteme as a relation territory and a place of multiple discourses. Besides, another aim is to link the mentioned inspection and the locator purpose and organizer of the notion of discourse with its functioning and institutionalization as well. This, in the context of the reflection on the political role of the intellectual, in which, thinking disciplines and trying to rebuild episteme, would be anyway a political function.

Keywords: Design, epistemology, discipline.

\section{Historia del discurso por historia del pensamiento}

Foucault se impone como tarea una reconstrucción de la historia a partir de reconstruir los saberes epistemológicos y sus sentidos en el ejercicio de sus relaciones, sus momentos fundacionales y sus objetos de estudio. No solamente en el texto particular que nos ocupa, sino mejor, en los referentes amplios de su ejercicio, esta reconstrucción de las instancias localizadoras de las particulares epistemes serán argumento y fuerza constitutiva para su saber, además de configurar el escenario donde aparecen algunos otros de sus objetos de estudio.

Para este caso particular, la tesis que propone y que le permite en principio dialogar con el propósito antes mencionado -la fundación epistemológica del diseño o, mejor, su condición disciplinar- es la de interrogar el sentido y valor del supuesto cambio como materia y fundamento de las transformaciones históricas y sustituir esta forma abstracta (cambio) por un análisis de los diferentes tipos de transformación.

El interés que presenta para nosotros se orienta a la posibilidad de rastrear un saber particular (diseño), no desde los tradicionales métodos históricos de las disciplinas modernas, dispuestas a partir del esfuerzo por localizar un momento original, dado a partir de las nociones de fundación y fundador, sino, en otro sentido, respondiendo más bien a la inspección de ciertas transformaciones que, miradas juiciosamente, podrían permitir la configuración de discursos que naveguen, antes que en territorios, en maneras, es decir, que tengan una forma antes que un lugar. Como excusa de trabajo sugerimos las transformaciones de las primeras décadas del siglo XX.

Por último, este texto quiere llamar la atención sobre el saber epistemológico como resultante de un proceso, bien de un acontecimiento o bien de una permanencia específica y no el acontecimiento, la permanencia o el proceso como resultantes de un saber epistemológico. 


\section{La forma del discurso}

El problema que se plantea Foucault, fundamental en su interés por determinar las fronteras disciplinares, es lo que él llama la Individualización de los discursos; aquello que lo ocupa es tratar de definir, en el discurso, los límites para reconocerlo como unidad; en ese sentido, no debería desbordarnos una pregunta del tipo: ¿es posible mencionar una unidad en el discurso del diseño?

Sujeto a este propósito, en principio, desecha dos recursos que han permitido la construcción del discurso: el histórico trascendental, que refiere a la fundación originaria, por ejemplo, aquel que refiere a que el discurso del Diseño se funda en la Inglaterra Victoriana y son las continuaciones ordenadas en el tiempo sus valores disciplinares; y el recurso empírico psicológico, referido al ejercicio hermenéutico a propósito de lo que quiso decir el fundador. Ejemplo de esto es el texto de Raymond Loewy (La laideur se vend mal. Ed.Gallimard. 1990), el cual se podría aceptar como fundación de un paradigma de autor. Bajo esta intención definitoria, el filósofo ordena una serie de enunciados metodológicos que tienen como objeto salvar la pregunta acerca de cómo se producen los discursos, cómo emergen estos ordenamientos. De tal manera que el método por él propuesto podría ser ordenado de la siguiente manera.

\section{a. Las características del discurso}

En un primer momento y con el objeto de detectar y sistematizar los caracteres propios de nuestros discursos, se sirve de tres criterios ordenadores, sobre los que yo haré referencia a partir de la historia del diseño, a manera de ejemplo: criterios de formación, sobre las reglas que han permitido la formación de objetos y conceptos; criterios de transformación, a partir de que han sido puestas en marcha nuevas reglas; y criterios de correlación, conjunto de relaciones que se definen y sitúan entre dos discursos (Foucault, 1991, pág. 50).
Podemos aquí servirnos de la emergencia de las vanguardias soviéticas del siglo XX, a manera de excusa, y en particular de los manifiestos construidos por estos grupos de vanguardia y la forma como permitieron la inserción de sus maneras y productos dentro del discurso de las artes y del diseño, e hicieron por esa vía las veces de justificaciones a los mismos y a sus formas de acceso al territorio del arte, pero también a ese nuevo que emergía, aún sin nombre, que entendíamos como el arte de la función.

Estas nuevas formas de argumentar la existencia de la obra en tanto producto del arte y del artista, transformaron el lugar preexistente además de enunciar desde otras perspectivas disciplinares las nuevas proposiciones discursivas. Bien sabidos son los casos del Constructivismo y el Productivismo respecto a sus vínculos con la fuerza transformadora del proletariado resuelto y convencido; o del Futurismo, con relación a los desarrollos de la ciencia y la tecnología; pero sin riesgo alguno podríamos señalar que los movimientos de vanguardia se alimentaron cada uno en sus particulares saberes de enunciados venidos de otros territorios disciplinares. Así, los criterios de formación, transformación y correlación pueden ser inspeccionados en las fuertes transformaciones y fundaciones disciplinares en las Artes Visuales y en las emergencias disciplinares en los primeros veinte años del siglo XX, entendidas como evidencia de las efervescentes sociedades que los propiciaron. La mirada epistemológica debería concentrarse en las transformaciones, no en los movimientos y tampoco en los resultados de sus procesos.

Los criterios anteriores permitirán sustituir los temas de la historia totalizante, ordenados de manera unidireccional y lineal, como una secuencia de acontecimientos, de maneras o de nombres, por análisis diferenciados y particulares de las tensiones políticas y sociales de los colectivos de creadores y productores. Además de describir tipos de historia y de ocuparse del sentido de la épistéme como un espacio abierto, fluctuante, en construcción y sobretodo como un espacio relacional. 


\section{b. Las transformaciones del discurso}

El propósito del método es el de "sustituir la forma abstracta, general y monótona del cambio a través de la cual se tematiza ingenuamente la sucesión, por el análisis de los tipos diferentes de transformación" (Foucault, 1991, pág. 52), lo que implica el análisis de las transformaciones, éstas que no generaron o provocaron el cambio sino que lo constituyen.

A los cambios que afectan a las formaciones discursivas mismas, Foucault los llama mutaciones; son cuatro básicamente: desplazamiento de las líneas que definen el objeto, nueva posición del sujeto en el discurso, nuevo funcionamiento del lenguaje en relación a los objetos, y nueva forma de circulación de los discursos. Volvamos a nuestro ejemplo de las vanguardias y acerquémonos particularmente a un ejemplo que resultará elemental, el tránsito de la escultura tradicional al ready- made dadaísta, en el cual al parecer podemos localizar una mutación respecto de las líneas que definen un ejercicio de escultura tradicional. En términos muy generales, digamos algunas cosas: el creador ya no es el ejecutor, los objetos son encontrados listos, así, el artista sólo los señala; es evidente que el lenguaje con el que debemos relacionarnos con estos objetos se ha también transformado y ni artistas, ni obras circulan en los lugares que habían estado determinados para las prácticas artísticas; se ven en la obligación de fundar nuevos territorios para dichas prácticas.

En lo que hoy entenderíamos por Diseño no hay evidentemente una mutación, en tanto no estaba establecida manera alguna de fundación epistemológica aceptada que pudiera ser transformada. Se acepta en principio una nueva posición del sujeto en el discurso, un nuevo funcionamiento del lenguaje y una nueva forma de circulación, pero no son nuevos respecto a algo que tuviera ya una existencia reconocida como epistemológica, por lo que a diferencia del mundo del arte, en diseño no podemos mencionar en términos precisos una mutación.
Por otra parte, los cambios que afectan a varias disciplinas al mismo tiempo, son cambios propios de la epistéme. A estos Foucault los llama redistribuciones. Son tres: alteración de las jerarquías, alteración de la relación y desplazamientos funcionales; aquí, quizá, la escuela de "la Bauhaus" -por centésima vez- pueda servirnos como ejemplo; fundada básicamente en los principios de la Arquitectura y el Arte, desde sus maneras y sus ejercicios pedagógicos. Con una fuerte orientación hacia la formación en oficios, pero sobre todo desde su mirada amplia sobre la creación, la escuela generó reordenamientos no solo en las disciplinas fundadoras (arte y arquitectura), sino que sin duda permitió el ordenamiento disciplinar de otros lugares como el Diseño Industrial y el Diseño Grafico, argumento fundacional de las academias. 
Además de generar nuevas relaciones entre las artes y mejor aún, entre la creación y la producción objetual, aquello que debemos señalar con precisión, es que si la escuela y sus continuaciones transformaron el horizonte del discurso disciplinar, entonces es claro que había antes algo que podía ser transformado, lo que sugiere pensar que la escuela es parte de un proceso que es posible rastrear conceptualmente y es precisamente allí en donde radicaría su importancia, en hacer evidente el posible levantamiento arqueológico del saber disciplinar en tanto discurso. Que los programas pedagógicos fundamentaran la aparición y el ejercicio académico del diseño, viniendo ellos de un cuerpo disciplinar diferente, haría entonces posible inspeccionar qué tipo de prácticas y qué tipo de dependencias definen. Dicho lo anterior, lo importante no sería la construcción de una tipología cerrada y lineal histórica, sino, por el contrario, intentar comprender la historia de las ideas disciplinares como el análisis de las transformaciones efectuadas, intentar describir los tipos de transformación de la práctica discursiva, además de entender el discurso como un lugar de posiciones y funcionamientos para los sujetos y definir allí las dependencias.

\section{c. La existencia del discurso}

Podemos entonces ya desprendernos de las reglas formales o de los códigos para reanudar el interés en los sucesos, la ley de existencia de los enunciados que se ordenará a partir de una Arqueología, como la descripción de un archivo y en este incluido el conjunto de sus reglas, reglas que deben definir los límites y las formas de decibilidad (¿de qué se puede hablar?), conservación (¿qué enunciados van a formar parte de la memoria de los hombres?), de memoria (¿qué relaciones existen entre los enunciados presentes y los pasados?), de reactivación (¿Qué discursos se intentan reconstruir?) y de la apropiación (¿Qué individuos tienen acceso al tipo de discursos? (Foucault, 1991, págs. 57-58)). Nuevamente vamos a los movimientos de vanguardia para ocuparnos, con un ejemplo, de los anteriores límites y formas.

Con respecto a la decibilidad, desde las transformaciones sucedidas en los movimientos, es claro sólo con respecto a lo que entendemos por artes visuales - como ya dijimos- que sobre lo que podemos hablar es distinto, por lo menos, a aquello sobre lo que antes podíamos hacerlo. No hablamos ahora, por ejemplo, solamente de escultura, sino también de ensamblajes, montajes y ready-mades, entre otros, y sin aún siquiera prever nociones insustituibles en este tiempo último como instalación y performance que derivan de las anteriores.

Respecto a lo que podemos decir en Diseño, no parece existir la misma claridad y en términos de producción seguimos hablando de objeto e inscritas en éste las ideas generales de función y forma. Sólo se percibe un cambio cuando nociones como interacción, situación, contexto e interpretación, o aquellas de dispositivo o evento, comienzan a hacer parte del horizonte propositivo de la disciplina, entrada ya la segunda mitad del siglo XX, pero no apropiadas particularmente por un saber disciplinar; lo que no hace posible señalar un momento de emergencia en tanto sólo se cumple uno de los requisitos. 
En relación a conservación y memoria, cabe la pregunta sobre cuáles enunciados van a formar parte de la memoria de quienes construyen el campo y qué relaciones existen entre los enunciados presentes y los pasados, o para cuáles sus formas y maneras entrarán a formar parte de la memoria disciplinar y de aquella propia de los sujetos que construyen la disciplina.

A propósito de la reactivación, parece claro que no todos los productos tenían la pretensión de inscribirse en la historiografía del arte pero no significa eso que pretendieran hacerlo en alguna otra. Es posible que una reflexión sobre como estos ejercicios de vanguardia, que apuntaban a lugares marginales de la práctica artística, hayan adquirido, luego de reacomodaciones de los lugares de artista, obra e institución, el lugar que correspondía a la obra de arte tradicional, donde sus formas de aparición estarían sujetas a los lugares tradicionales de colección, galería y museo y también hayan hecho el tránsito a territorios no colonizados por el arte; pero habrá que insistir en que no es posible saber qué otra disciplina se intenta reconstruir.

Apropiación, por el contrario es un criterio sobre el cual va a ser posible instaurar evidencias disciplinares, en tanto que lenguaje y comunidad comienzan a especializarse en la disciplina.

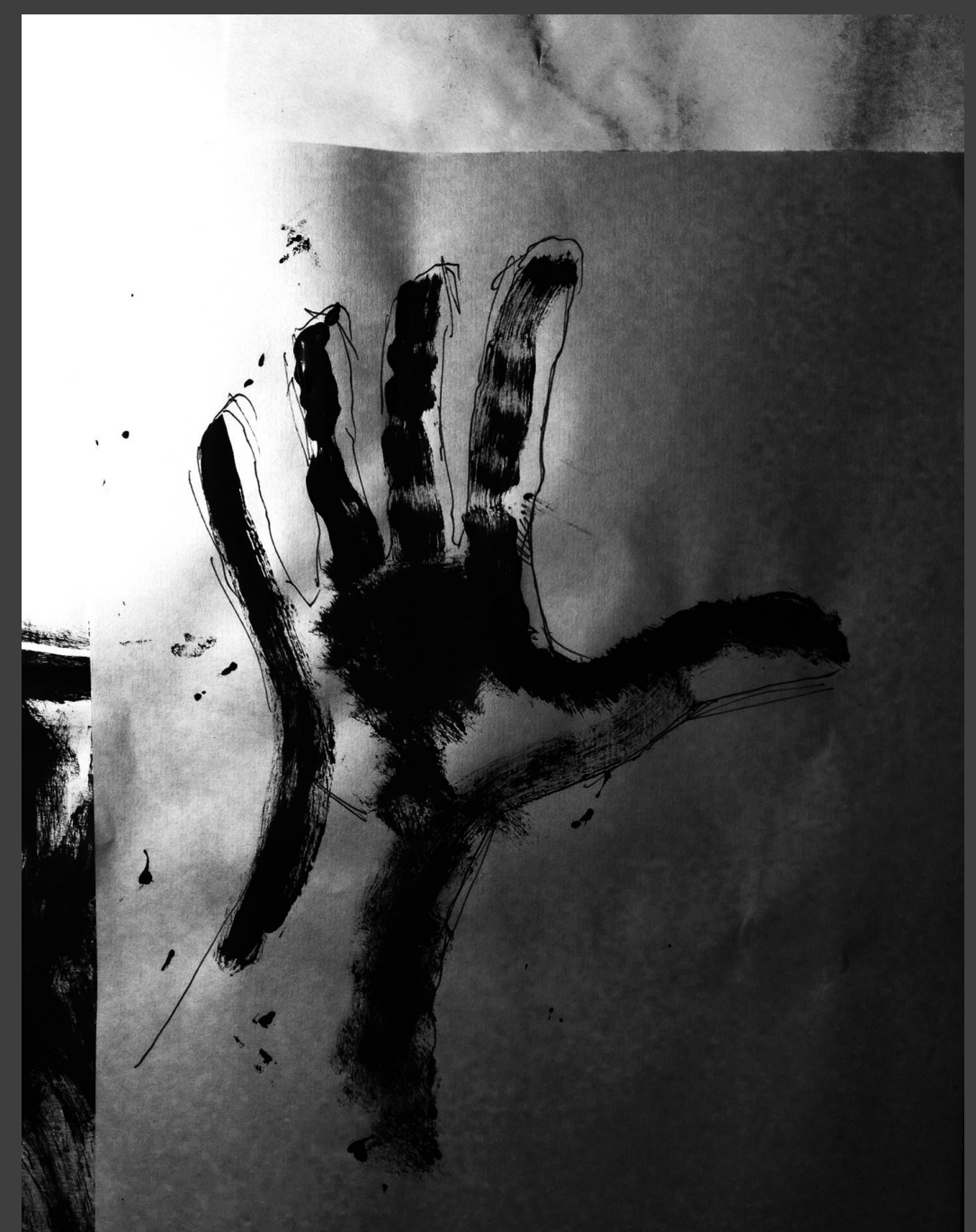


A partir de lo anterior, tres importantes consecuencias señala Foucault: tratar el discurso pasado no como un comentario sino como un monumento, buscar en los discursos sus leyes de existencia y referir el discurso al campo en el que se despliega. (Foucault, 1991, pág. 59).

Para concluir la mirada y reflexión sobre el método, Foucault sugiere abordar luego las relaciones entre lo que se dice y una práctica política desde las operaciones críticas; apunta particularmente como un problema el del estatuto de los discursos científicos, asumiendo que la práctica política ha transformado no el sentido ni la forma del discurso sino sus condiciones de emergencia, de inserción y de funcionamiento. Las operaciones críticas serían:

1. Poner en duda la idea de que en el discurso se pueden establecer los límites.

2. Borrar las oposiciones poco pensadas, dicotomías antiguo- nuevo, vivo-muerto y liberar a la historia de la triple metáfora, evolucionista, biológica y dinámica.

3. Suprimir la negación que ha afectado al discurso, desde tres argumentos, tratar el discurso como lugar de expresión, reconocer en el discurso los segmentos de tipo psicológico, lingüístico y semántico y admitir que todas las operaciones están hechas fuera del discurso.

4. Presentar el análisis del discurso desde sus condiciones de formación y desde las relaciones que hace posibles, con el objeto de enfrentarnos ya no a una historia de las ideas sino a una historia de las prácticas discursivas (Foucault, 1991, 62-64). Así, la ruptura en la tradición sugerida por los movimientos de vanguardia no es la única que ha sucedido en la construcción epistemológica del diseño, luego el discurso de la disciplina del diseño no puede establecer los límites del discurso mismo ni aquellos del arte. Lo que permite pensar que una localización disciplinar ha de estar construida desde la invitación metódica de Foucault, que debe ser mirada con juicio y dispuesta con rigor para proponer una reconstrucción eventual de la disciplina del Diseño, es decir, el método sugiere sin duda una manera de construcción distinta de los saberes particulares y de las epistemes, donde la construcción disciplinar, podría ser una construcción de los propósitos sobre los cuales reflexionar.

Por último, en el proceso metódico que propone Foucault, habría que señalar que el filósofo afirma que la práctica política ha transformado, no el sentido ni la forma del discurso, sino su modo de existencia y su sistema de formación, el sistema mismo de formación de los conceptos; asunto de importancia mayor, en lo que refiere al enunciado primero de la función política y como esta cobra sentido en la configuración e instauración de un discurso o en el hecho mismo de pensar la disciplina, o lo que debemos reconstruir para imaginarla. 
